\title{
Análisis psicométrico y adaptación de la Escala de Distancia Social (DS) en una muestra chilena
}

\author{
Pamela Grandón Fernández, ${ }^{1}$ Félix Cova Solar, ${ }^{1}$ Sandra Saldivia Bórquez, ${ }^{2}$ Claudio Bustos Navarrete
}

Artículo original

\section{ABSTRACT}

\section{Background}

Stigma is one of the major impairments to social integration of people with mental disorders. The present research was aimed to study the psychometric properties and adapt the Link's Social Distance Scale (SD) in a sample from Chile. This scale assessed the public attitudes toward people with mental disorders by means of a vignette, followed by a set of questions.

\section{Method}

A total of two samples by convenience were taken. The first sample consisted of 399 people and the second of 350 . Reliability was assessed by means of the Cronbach's alpha coefficient. In order to validate its structure, an exploratory factor analysis of the first sample was conducted. Like waise, a confirmatory analysis was conducted with the second sample. Finally, the Pearson correlation coefficient was used to study the concurrent validity of the Orientation Scale to Social Dominance.

\section{Results}

Results indicate that the final instrument has two factors "proximity and social interaction," composed by three items, and "intimacy and confidence," composed by two. Despite the fact that the samples had different educational level, the adjustment indixes obtained from the confirmatory analyses, as well as the internal consistency of the instrument, were appropriate.

\section{Discussion and conclusion}

The existence of two factors can be related to the growth of the community mental health model in Chile. This has produced an impact on the amount of community centers and, therefore on the closeness on the subject. The results obtained support the possibility of using this instrument in different social groups.

Key words: Social stigma, mental disorders, validation studies.

\section{RESUMEN}

\section{Antecedentes}

El estigma es uno de los obstáculos principales para la integración social de las personas con trastornos mentales.

\section{Objetivo}

Estudiar las propiedades psicométricas y adaptar la Escala de Distancia Social de Link (DS) en una muestra chilena. Ésta evalúa las actitudes públicas hacia personas con trastornos mentales mediante una viñeta seguida de un grupo de preguntas.

\section{Método}

Se tomaron dos muestras por conveniencia: la primera estuvo compuesta por 399 personas y la segunda por 350. La confiabilidad se evaluó por medio del coeficiente alfa de Cronbach. Para valorar su estructura factorial, se realizó un análisis factorial exploratorio con la primera muestra y uno confirmatorio con la segunda. Finalmente, se utilizó el coeficiente de correlación de Pearson para estudiar la validez concurrente con la Escala de Orientación a la Dominancia Social.

\section{Resultados}

Los resultados indican que el instrumento final posee dos factores "cercanía e interacción social", compuesto por tres reactivos, e "intimidad y confianza", compuesto por dos. A pesar de que las muestras tenían distinto nivel educativo, los índices de ajuste del modelo obtenido en el análisis factorial confirmatorio, así como la consistencia interna del instrumento, fueron adecuados.

\section{Discusión y conclusión}

La existencia de dos factores puede estar relacionada con el crecimiento del modelo de salud mental comunitaria en Chile, lo que ha impactado en la cantidad de dispositivos sanitarios y por tanto en la cercanía que se tiene sobre el tema. Los resultados obtenidos avalan la posibilidad de usar este instrumento en distintos grupos sociales.

Palabras clave: Estigma social, trastornos mentales, estudios de validación.

Departamento de Psicología, Facultad de Ciencias Sociales, Universidad de Concepción, Concepción, Chile.

2 Departamento de Psiquiatría y Salud Mental, Facultad de Medicina, Universidad de Concepción, Concepción, Chile.

Correspondencia: Pamela Grandón Fernández. Departamento de Psicología, Facultad de Ciencias Sociales, Universidad de Concepción, Barrio Universitario s/n, Concepción, Región del Biobio, Chile. E-mail: pgrandon@udec.cl

Recibido: 3 de abril de 2014. Aceptado: 16 de julio de 2014 


\section{ANTECEDENTES}

Los trastornos mentales graves (TMG) generan un importante sufrimiento personal y social que deriva no sólo de sus síntomas, sino del estigma asociado a ellos. ${ }^{1} \mathrm{El}$ término estigma alude al proceso de etiquetamiento, pérdida de estatus y discriminación de la que es objeto una persona que presenta un atributo evaluado como negativo por su comunidad. ${ }^{2}$ Entre las consecuencias directas del estigma destaca la visión que tiene el propio sujeto de sí mismo como incapaz, lo que genera sentimientos de vergüenza y favorece el aislamiento. Por otra parte, se asocia con una gravedad de los síntomas y una mala adherencia al tratamiento, lo que aumenta el riesgo de recaídas. ${ }^{3}$ Diversas investigaciones han constatado que la presencia de trastornos mentales graves se vincula a consecuencias discriminatorias en el acceso al empleo y la vivienda, así como a distintos tipos de relaciones sociales significativas como la pareja y los amigos. ${ }^{4}$

Hay abundante evidencia del efecto de barrera que cumple el estigma en el acceso a los servicios sanitarios: las personas rechazan usar los servicios de salud mental para evitar el prejuicio y la discriminación asociados a tener un diagnóstico psiquiátrico. En Chile, la falta de conocimiento acerca de las enfermedades mentales y el estigma constituyen las principales razones por las que la población no busca tratamiento. ${ }^{5}$

El estigma se ha evaluado a partir de la opinión de los propios afectados y del público general. En el primer caso, se evalúan la percepción, las experiencias y el autoestigma en personas con TMG. ${ }^{6}$ En tanto, en el segundo, se han estudiado las creencias y actitudes de la población hacia las personas con diagnóstico psiquiátrico. ${ }^{7}$ Este tipo de estigma denominado "público" es relevante pues se relaciona con las conductas discriminatorias, basadas en prejuicios y estereotipos, hacia este grupo social. ${ }^{8}$

Se han utilizado distintos tipos de instrumentos para evaluar el estigma público en población adulta. Aquí destacan las escalas de opinión sobre los trastornos mentales, las escalas atribucionales, las escalas de reacciones emocionales y las escalas de distancia social. ${ }^{9}$ Estas últimas miden la voluntad de los encuestados para interactuar con una persona determinada en un tipo de relación específica. Estas escalas buscan que los encuestados señalen su rechazo o aceptación hacia una persona en particular a partir de la presentación de una viñeta. Estos instrumentos poseen la ventaja de ser cortos y medir las actitudes con base en una situación hipotética, pero real, lo que los aproxima más a la conducta que podría tener una persona al relacionarse con otra que presente un TMG. En general, presentan una excelente consistencia interna y se han utilizado en distintos contextos. ${ }^{9}$ Dentro de las escalas de distancia social, la más utilizada ha sido la Escala de Distancia Social de Link, ${ }^{10}$ que proporciona una descripción hipotética bien detallada de una persona con trastorno mental grave (viñeta), en relación con la cual el encuestado debe responder una serie de preguntas.
Los escasos estudios realizados en Chile sobre el tema indican que existen prejuicios y actitudes negativas hacia las personas con diagnóstico psiquiátrico. Estas investigaciones han utilizado instrumentos de elaboración propia o traducida, pero no adaptados al contexto nacional. ${ }^{11,12} \mathrm{Si}$ consideramos que la modificación del estigma hacia personas con TMG constituye un desafío relevante desde el punto de vista de la salud pública, que tendría impacto en la calidad de vida de estas personas, contar con instrumentos que permitan evaluarlo es de gran importancia.

\section{OBJETIVO}

Estudiar las propiedades psicométricas y adaptar la Escala de Distancia Social de Link. Se evaluó su confiabilidad, su estructura factorial y su validez concurrente con la escala de orientación a la dominancia social.

\section{MÉTODO}

\section{Muestra}

Se tomaron dos muestras por conveniencia, no probabilísticas, de población general radicada en el área del gran Concepción. Las personas fueron reclutadas en lugares de gran afluencia de público: terminal de buses, salas de espera de recintos sanitarios, municipios, plazas, universidades y supermercados, y en distintos barrios del territorio seleccionado. La primera estuvo constituida por 399 personas y la segunda, por 350. El único criterio de inclusión fue tener entre 18 y 65 años.

\section{Descripción de las muestras}

La distribución de hombres y mujeres en ambas muestras fue similar, siendo las segundas el grupo mayoritario con más de $55 \%$ del total. La media de edad fue ligeramente mayor en la segunda muestra, donde alcanzó 41.9 años por sobre los 39.1 años de la primera. Un 52.1\% (208) de la primera muestra estaba casado y convivía, en tanto que en la segunda este porcentaje subió a 62\% (217). Respecto al nivel educacional, la mayoría de los sujetos tenían enseñanza media, ya fuera completa o incompleta. Sin embargo, en la primera muestra el porcentaje fue menor (38.1\%) que en la segunda (59.4\%). Por otra parte, en la primera muestra hubo un porcentaje mayor de personas que poseían enseñanza universitaria $(29.6 \%)$ que en la segunda (4.3\%). En las dos muestras, la mayoría de los sujetos trabajaba (68.8 y 63.1\%, respectivamente). Al consultárseles si tenían un pariente con un trastorno mental grave, los porcentajes de ambas muestras fueron similares: 19 y $17.1 \%$. Sin embargo, en la segunda muestra un porcentaje más alto de los participantes señaló tener contacto con el familiar referido (78.3 vs. $68.4 \%$ ). En relación con los veci- 
Cuadro 1. Características sociodemográficas de las muestras

\begin{tabular}{|c|c|c|}
\hline Variable & $\begin{array}{c}\text { Primera muestra } \\
\qquad(n=399)\end{array}$ & $\begin{array}{c}\text { Segunda muestra } \\
(n=350)\end{array}$ \\
\hline \multicolumn{3}{|l|}{ Género } \\
\hline Masculino & 177 (44.4\%) & 155 (44.3\%) \\
\hline Femenino & $222 \quad(55.6 \%)$ & 195 (55.7\%) \\
\hline Total & $399(100.0 \%)$ & $350(100.0 \%)$ \\
\hline \multicolumn{3}{|l|}{ Edad } \\
\hline Media & 39.13 & 41.91 \\
\hline Desv. Típ. & 12.57 & 12.57 \\
\hline Rango & $(18-65)$ & $(18-65)$ \\
\hline \multicolumn{3}{|l|}{ Estado civil } \\
\hline Casado/conviviente & $208 \quad(52.1 \%)$ & $217 \quad(62.0 \%)$ \\
\hline Soltero & $153 \quad(38.3 \%)$ & 89 (25.4\%) \\
\hline Viudo/separado & $38 \quad(9.6 \%)$ & $44 \quad(12.6 \%)$ \\
\hline Total & $399(100.0 \%)$ & $350(100.0 \%)$ \\
\hline \multicolumn{3}{|l|}{ Nivel educacional } \\
\hline Sin escolaridad & $(0.0 \%)$ & $(0.3 \%)$ \\
\hline Básica & $35 \quad(8.8 \%)$ & $46 \quad(13.1 \%)$ \\
\hline Media & $152 \quad(38.1 \%)$ & $208 \quad(59.4 \%)$ \\
\hline Técnica & $94 \quad(23.5 \%)$ & $80 \quad(22.9 \%)$ \\
\hline Universitaria & $118 \quad(29.6 \%)$ & $15 \quad(4.3 \%)$ \\
\hline Total & $399(100.0 \%)$ & $350(100.0 \%)$ \\
\hline \multicolumn{3}{|l|}{ Años escolaridad } \\
\hline Media & 6.10 & 4.97 \\
\hline Desv. Típ. & 2.19 & 1.50 \\
\hline Rango & $(2-9)$ & $(1-9)$ \\
\hline \multicolumn{3}{|l|}{ Ocupación actual } \\
\hline Estudiante & $46 \quad(11.5 \%)$ & $19 \quad(5.4 \%)$ \\
\hline Dueña de casa & $45 \quad(11.3 \%)$ & $76 \quad(21.8 \%)$ \\
\hline Trabajador & $274 \quad(68.8 \%)$ & $221 \quad(63.1 \%)$ \\
\hline Cesante & $17 \quad(4.2 \%)$ & $15 \quad(4.3 \%)$ \\
\hline Jubilado & $17 \quad(4.2 \%)$ & $19 \quad(5.4 \%)$ \\
\hline Total & $399(100.0 \%)$ & $350(100.0 \%)$ \\
\hline \multicolumn{3}{|l|}{ Pariente con TMG } \\
\hline Pariente con TMG & $76 \quad(19.0 \%)$ & $60 \quad(17.1 \%)$ \\
\hline Pariente sin TMG & $323 \quad(81.0 \%)$ & $290 \quad(82.9 \%)$ \\
\hline Total & $399(100.0 \%)$ & $350(100.0 \%)$ \\
\hline \multicolumn{3}{|l|}{ Contacto con pariente } \\
\hline Mantiene contacto & $52 \quad(68.4 \%)$ & $(78.3 \%)$ \\
\hline No mantiene contacto & $24 \quad(31.6 \%)$ & $13 \quad(21.7 \%)$ \\
\hline Total & $76 \quad(100.0 \%)$ & $60(100.0 \%)$ \\
\hline \multicolumn{3}{|l|}{ Vecino con TMG } \\
\hline Vecino con TMG & $101 \quad(25.3 \%)$ & $71 \quad(20.3 \%)$ \\
\hline Vecino sin TMG & $298 \quad(74.7 \%)$ & $279 \quad(79.7 \%)$ \\
\hline Total & $399(100.0 \%)$ & $350(100.0 \%)$ \\
\hline \multicolumn{3}{|l|}{ Contacto con vecino } \\
\hline Mantiene contacto & $70 \quad(69.3 \%)$ & $45 \quad(63.4 \%)$ \\
\hline No mantiene contacto & $31 \quad(30.7 \%)$ & $26 \quad(36.6 \%)$ \\
\hline Total & $101(100.0 \%)$ & $71(100.0 \%)$ \\
\hline
\end{tabular}

nos, ocurrió lo contrario: las personas de la primera muestra señalaron tener mayor frecuencia de contacto que los de la segunda (69.3 y 63.4\%) (cuadro 1$)$.

\section{Instrumentos}

\section{Escala de Distancia Social (DS)}

Este instrumento fue desarrollado por Link et al. ${ }^{10}$ con base en la escala de Bogardus. ${ }^{13}$ Evalúa las actitudes públicas hacia las personas con trastornos mentales graves y está compuesto por una viñeta que expone el caso de una persona con un trastorno mental, seguido de siete reactivos en un formato de respuesta tipo Likert con cinco alternativas, que van desde nada de acuerdo a totalmente de acuerdo. Las preguntas indagan sobre distintas situaciones que varían en el grado de cercanía con el afectado. Entre éstas se cuentan ser vecino, amigo, empleado y pareja de una persona con un TMG. En nuestro estudio, se agregó al instrumento original una pregunta más general sobre la disposición a conversar con alguien que presente un TMG, por lo que el cuestionario quedó compuesto por ocho reactivos. Además, se elaboró una viñeta sobre una persona con esquizofrenia que se adaptara al contexto local. El cuestionario original obtuvo un coeficiente de confiabilidad de $\alpha=0.85 .{ }^{10}$

\section{Escala de Orientación a la Dominancia Social (SDO)}

Esta escala es una adaptación para población chilena de la escala original de Pratto, Sidanius, Stallworth y Malle. ${ }^{14}$ Se utilizó como indicador de validez concurrente, dado que presenta una correlación consistente con actitudes discriminatorias. La escala tiene dos factores: oposición a la igualdad y orientación a la dominancia, cada uno de los cuales comprende ocho reactivos. El formato de respuesta es tipo Likert con siete alternativas, que van desde totalmente en desacuerdo hasta totalmente de acuerdo. Presenta una elevada consistencia interna $(\alpha=0.86)$, con valores de $\alpha=0.79$ para la subescala oposición a la igualdad y de $\alpha=0.88$ en la de orientación a la dominancia. ${ }^{15}$

\section{Datos sociodemográficos}

Para recolectar la información sociodemográfica relevante, se elaboró un breve cuestionario que preguntaba por información de cada sujeto, incluyendo edad, género, estado civil, nivel educativo y actividad laboral. Además, se consultó por el contacto previo con personas con trastornos mentales graves, ya fueran familiares o vecinos.

\section{Procedimiento}

Se utilizó la traducción de cinco preguntas de la DS realizada en España. ${ }^{16}$ Las otras dos preguntas se tradujeron y retradujeron (inglés-español). Posteriormente, se adaptó el lenguaje y se elaboró una viñeta adicional basada en una 
situación tipo del contexto nacional. Previo a su aplicación definitiva, se hizo una aplicación piloto del instrumento en una muestra de 10 personas, cuya información no fue incorporada a los análisis posteriores.

La aplicación de los instrumentos fue conducida por estudiantes de psicología especialmente capacitados. Los participantes fueron reclutados como voluntarios y daban fe de esta voluntariedad al firmar un consentimiento informado. La mayoría contestó los cuestionarios de manera autoaplicada. Cuando un sujeto presentaba dificultades para responder, un entrevistador aplicaba los cuestionarios. En la primera muestra, la aplicación se realizó entre noviembre y diciembre de 2011, y en la segunda, entre diciembre de 2012 y enero de 2013.

\section{Análisis estadísticos}

Para evaluar la estructura factorial, se realizó un análisis factorial exploratorio sobre la primera muestra, cuya solución se probó mediante un análisis confirmatorio, realizado sobre la segunda muestra.

En el análisis factorial exploratorio se utilizó un método iterativo para determinar el número de factores iniciales posibles de extraer del conjunto de reactivos originales. Como procedimiento se empleó el Análisis Paralelo de Horn, basado en un remuestreo no paramétrico de 1000 muestras bootstrapping. ${ }^{17}$ Una vez determinado el número de factores, se generó una solución utilizando como método de extracción de factores el método de mínimos cuadrados ponderados sobre la matriz de correlaciones policóricas, seguido de una rotación oblicua Promax.

A partir de la solución factorial inicial, se consideraron asignados a un factor los que mostraran un coeficiente de configuración igual o mayor a 0.30 en algunos de los factores y que no incluyeran al cero dentro del intervalo de confianza del boots-trapping no paramétrico al 90\% para el factor considerado. Si se cumplían estos dos criterios para más de un factor, se asignaba el reactivo al factor donde su carga factorial fuera mayor si al menos duplicaba las cargas inferiores. A partir de los reactivos y factores resultantes de la primera etapa, se generaba una nueva solución factorial, repitiéndose el proceso de extracción de factores, rotación y selección de reactivos/factores, hasta encontrar una solución estable.

Los análisis de confiabilidad se llevaron a cabo mediante el coeficiente alfa de Cronbach. La validez de criterio concurrente se estimó con el coeficiente de correlación producto-momento de Pearson.

La información se codificó y procesó usando los paquetes estadísticos Stata 12, Mplus 7.0 y R 2.15.2.

\section{RESULTADOS}

El análisis factorial exploratorio arrojó dos soluciones. El análisis Very Simple Structure (VSS), ${ }^{18}$ mostró una solución
Cuadro 2. Matriz de coeficientes de configuración

\begin{tabular}{|c|c|c|c|}
\hline \multirow[b]{2}{*}{$\mathrm{N}^{\circ}$} & \multirow[b]{2}{*}{ Reactivos } & \multicolumn{2}{|c|}{ Factores } \\
\hline & & 1 & 2 \\
\hline 1 & $\dot{\partial}$ Trabajarías con una persona como Juan? & 0.44 & 0.33 \\
\hline 2 & $\begin{array}{l}\text { ¿Aceptarías como vecino a una persona } \\
\text { como Juan? }\end{array}$ & 0.82 & -0.05 \\
\hline 3 & ¿Serías amigo de una persona como Juan? & 0.93 & -0.15 \\
\hline 4 & $\begin{array}{l}\text { Si tuvieses una vivienda; } 2 \text { se la arrendarías a } \\
\text { alguien como Juan? }\end{array}$ & 0.30 & 0.50 \\
\hline 5 & $\begin{array}{l}\text { Si tuvieses un negocio, ¿darías trabajo a al- } \\
\text { guien como Juan? }\end{array}$ & 0.36 & 0.41 \\
\hline 6 & $\begin{array}{l}\text { ¿Tendrías como pareja a alguien como } \\
\text { Juan? }\end{array}$ & -0.14 & 0.86 \\
\hline 7 & $\begin{array}{l}\text { Si tuvieses hijos, ¿aceptarías que cuidara de } \\
\text { ellos una persona como Juan? }\end{array}$ & -0.17 & 0.87 \\
\hline 8 & 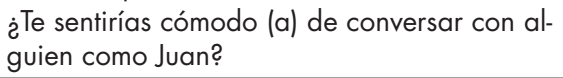 & 0.75 & -0.07 \\
\hline
\end{tabular}

de un factor, en tanto que el análisis paralelo de Horn mostró una solución de dos factores. La solución basada en un factor no cumplía con los criterios antes mencionados, por lo que se reemplazó por la solución de dos factores, que explica $57 \%$ de la varianza (cuadro 2).

El factor 1, que denominamos "Cercanía e interacción so-

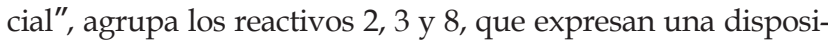
ción a aceptar una relación con una persona con un TMG, en una gradiente desde mayor cercanía (amistad) hasta conversar con alguien. El factor 2 corresponde a respuestas que indican una mayor confianza e intimidad con la persona, razón por la cual le llamamos "Intimidad y confianza", y está compuesto por los reactivos 6 y 7 . Se observa una correlación positiva entre ambos factores, $r=0.645$, lo que indica que una actitud de aceptación hacia las personas con un TMG se relaciona con el establecimiento de una relación de intimidad y confianza.

Con la segunda muestra de 350 personas se probó el modelo de dos factores mediante un análisis factorial confirmatorio. Los resultados del análisis factorial confirmatorio muestran un ajuste moderadamente bueno de los datos al modelo. Si bien el indicador de ajuste absoluto Chi-Cuadrado muestra que los datos no se ajustan completamente al modelo, $\chi^{2}(4)=12.372$, $p=0.0148$, esto es esperable en función del tamaño de la muestra. El índice CFI (Comparative Fit Index) es de 0.995, en tanto que el Chi-cuadrado normalizado es de 3.093, lo que se considera regular. Por otra parte, el TLI (Tucker-Lewis Index) alcanza un 0.987, lo que es adecuado, al igual que el RMSEA (Root Mean Square error of Approximation), que es de 0.077 (cuadro 3).

Cuadro 3. Indicadores de ajuste del análisis confirmatorio

\begin{tabular}{lc}
\hline Indicador & Valor \\
\hline Prueba de bondad de ajuste & $\chi^{2}(4)=12.372$ \\
Chi-cuadrado normalizado & 3.093 \\
RMSEA & 0.077 \\
CFI & 0.995 \\
TLI & 0.987 \\
\hline
\end{tabular}




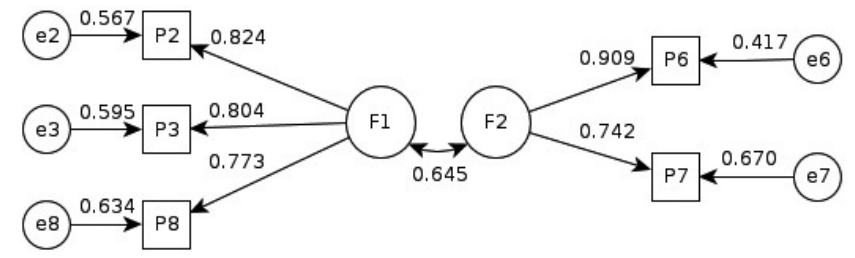

Figura 1. Estimadores de los parámetros del modelo.

En la figura 1 se presentan los estimadores para los parámetros del modelo. Se puede ver que todas las correlaciones entre los factores y los reactivos son significativas, y el valor absoluto de todas las cargas se ubica sobre el valor de 0.5 recomendado por Hair et al. ${ }^{19}$

Para el factor 1 , la varianza extraída es de 0.64 , con una validez de constructo de 0.94 , en tanto que el factor 2 presenta una varianza extraída promedio de 0.69 y una validez de constructo de 0.90 , lo que representa un muy buen ajuste de los reactivos a los factores.

\section{Confiabilidad}

La consistencia interna de cada uno de los factores alcanzó valores de alfa de Cronbach de 0.82 para el factor 1 y de 0.75 para el factor 2 , y de 0.78 para la escala total.

\section{Validez concurrente}

En el cuadro 4 se indican las correlaciones entre los dos factores de la DS adaptada y la escala SDO. Como se puede ver, todas las correlaciones son negativas y casi todas son significativas, a excepción del factor 2 de la DS y el factor 2 de la ODS. La mayor correlación se da entre el factor 1 de la DS, que indica una relación con la cercanía y la interacción social con personas con TMG, y el factor 1 de la SDO, que va de la oposición a la igualdad.

\section{DISCUSIÓN Y CONCLUSIÓN}

El propósito de esta investigación fue estudiar las propiedades psicométricas de la Escala de Distancia Social (DS) destinada a evaluar las actitudes del público general hacia las personas con trastornos mentales. Este instrumento tiene la

Cuadro 4. Correlaciones producto-momento de Pearson entre los factores de la escala DS y la Escala de Orientación a la Dominancia Social (SDO)

\begin{tabular}{lccc}
\hline & Factor 1 SDO & Factor 2 SDO & SDO Total \\
\hline Factor 1 DS & $-0.246^{* * *}$ & $-0.1585^{* * *}$ & $-0.258^{* * *}$ \\
Factor 2 DS & $-0.189 * * *$ & -0.0937 & $-0.180^{* * *}$ \\
DS Total & $-0.260 * * *$ & $-0.155^{* * *}$ & $-0.265^{* * *}$ \\
\hline
\end{tabular}

${ }^{* *}: p<0,01 ;{ }^{* *}: p<0,001$. ventaja de ser breve y puede aplicarse a poblaciones diversas, más allá de aquéllas en estrecho contacto con personas con diagnóstico psiquiátrico. Asimismo, por estar basado en preguntas sobre un caso real, sus respuestas se acercan más a la intención conductual de los consultados. Por otra parte, la diversidad de muestras usadas en el análisis psicométrico y la convergencia de sus resultados es un indicador que da mayor validez al uso del instrumento en distintos grupos sociales. En cuanto a la confiabilidad, los análisis indican que el instrumento alcanza un nivel de consistencia aceptable para su empleo en población general.

La estructura factorial de la escala mostró diferencias respecto del instrumento original. La escala norteamericana posee un factor que agrupa al conjunto de reactivos; en el presente estudio se identificaron dos factores: "Cercanía e interacción social", con tres reactivos, e "Intimidad y confianza", con dos. Los reactivos eliminados presentaban una carga cruzada que no permitía incluirlos de manera confiable en ninguno de los factores. Destaca que estos reactivos corresponden a preguntas sobre trabajo y vivienda, es decir, sobre la apertura a la inclusión social de las personas con TMG. En tanto, las preguntas que saturaron de manera clara en uno de los dos factores están más relacionadas con una interacción cercana e íntima con el afectado. Al parecer, en el contexto local, favorecer la inclusión de estas personas no se asocia exclusivamente a un tipo de relación (más o menos personal), sino que puede expresarse de manera indistinta en alguna de ellas. Por otra parte, existen diferencias entre relaciones de cercanía y de confianza e intimidad dadas por la configuración de estos dos factores.

La distinción de estas dimensiones puede estar asociada a que en Chile se ha incrementado la cantidad de dispositivos de salud mental en la comunidad, lo que deriva del fortalecimiento del modelo de salud mental comunitaria en la última década. ${ }^{20}$ En este contexto, las personas están más informadas sobre el tema y, como resultado de este aumento, puede que tiendan a ver como más posible el contacto con personas con TMG, lo que a su vez podría llevar a establecer una diferenciación más fina sobre el tipo de relación en que estarían dispuestos a involucrarse con un usuario del programa de salud mental.

Existían algunas diferencias sociodemográficas entre ambas muestras, lo que puede haber influido en que no fueran más elevados los indicadores del AFC. La primera era más educada y el grupo de personas con estudios técnicos y universitarios suman más de $50 \%$ del total, mientras que en la segunda sólo alcanzaban 25\%. Diversas investigaciones señalan que el nivel educativo es una variable que, consistentemente, se asocia con las actitudes hacia las personas con trastornos mentales. Los sujetos más educados tienden a mostrar actitudes más favorables que las personas con menos escolaridad, ${ }^{21-23}$ diferencia que puede influir en la diversidad de actitudes de los grupos, por lo que es más difícil que se logre un ajuste exacto entre ambas. Pese a ello, los ni- 
veles de ajuste obtenidos son apropiados, e indican la posibilidad de usar el instrumento en distintos grupos sociales.

Dado que existe evidencia de que el nivel de contacto con personas con un trastorno mental influye en las actitudes hacia éstas, ${ }^{21,24,25}$ se preguntó por esta variable. Destaca que, aunque existe un porcentaje minoritario de personas que poseen un familiar o vecino con un TMG, aquellos que lo tienen mantienen mayoritariamente contacto con éste.

Se observó asimismo una convergencia entre casi todas las dimensiones evaluadas por la DS con los valores de la ODS. La mayor correlación se obtuvo entre los totales de ambas escalas, toda vez que actitudes autoritarias y de rechazo hacia determinados grupos sociales se asocian negativamente con el establecimiento de relaciones cercanas y de confianza con estas personas.

Una de las limitaciones del estudio es su muestreo no probabilístico, lo que obliga a tener cautela al generalizar los resultados. Por otro lado, no se consideraron otros indicadores de confiabilidad, en particular la confiabilidad testretest que permite dar cuenta de la estabilidad temporal de las medidas.

En el futuro sería necesario realizar investigaciones que indaguen y ahonden sobre cómo se presenta el estigma entre el público nacional pues, de acuerdo con resultados de otros estudios en el área, en Chile existen actitudes negativas hacia las personas con estas características. Esto permitiría avanzar en la elaboración de programas que disminuyan estas actitudes y que por tanto favorezcan la inclusión social de estos sujetos.

\section{Financiamiento}

Esta investigación fue posible gracias al financiamiento de la Dirección de Investigación de la Universidad de Concepción, Proyecto "Validación de la entrevista de Evaluación Integral de los Estados Mentales en Riesgo", DIUC No 209.083.039-1.0. El proyecto se ejecutó entre marzo de 2011 y julio de 2013.

\section{Conflicto de intereses}

Los autores declararon no tener conflicto de intereses.

\section{REFERENCIAS}

1. Arboleda-Flórez J. Stigma and discrimination: an overview. World Psychiatry 2005;4:8-10.

2. Link B, Phelan J. Conceptualizing stigma. Annual Review Sociology 2001;27:363-385.

3. Livingston JD, Boyd J. Correlates and consequences of internalized stigma for people living with mental illness: A systematic review and meta-analysis. Soc Sci Med 2010;71:2150-2161.

4. Sharac J, Mccrone P, Clement S, Thornicroft G. The economic impact of mental health stigma and discrimination: A sistematic review. International J Epidemiology Psychiatric Sciences 2010;19:223-232.

5. Vicente V, Kohn R, Saldivia S, Rioseco P. Carga del enfermar psíquico, barreras y brechas en la atención de salud mental en Chile. Rev Med Chil 2007;135:1591-1599.

6. Brohan E, Slade M, Clement S, Thornicroft G. Experiences of mental illness stigma, prejudice and discrimination: a review of measures. BMC Health Services Research 2010;10:80.

7. Corrigan PW, O`Shaughnessy JR. Changing mental illness stigma as it exists in the real world. Australian Psychologist 2007;42:90-97.

8. Corrigan PW, Shapiro JR. Measuring the impact of programs that challenge the public stigma of mental illness. Clin Psycho Rev 2010;30:907-922.

9. Link B, Yang L, Phelan J, Collins P. Measuring mental illness stigma. Schizophr Bull 2004;30:511-541.

10. LinkB, Cullen FT, Frank J, Wozniak JF. The social rejection of former mental patients. Understanding why label matter. American sociological Review 1987;54:100-123

11. Chuaqui J. Esquizofrenia, estigma e inserción laboral. Psiquiatría Salud Mental 2002;1:4-11.

12. Zárate $C$, Ceballos $M$, Contardo $M$, Florenzano R. Influencia de dos factores en la percepción hacia los enfermos mentales; contacto cercano y educación en salud. Rev Chil Neuro-Psiquiat 2006;44:205-214.

13. Bogardus ES. Social distance and its origins. J Applied Sociology 1925;9:216-226.

14. Pratto F, Sidanius J, Stallworth L, Malle B. Social dominance orientation: A personality variable predicting social and political attitude. $\mathrm{J}$ Pers Soc Psycho 1994;67:741-763.

15. Cárdenas M, Meza $P$, Lagues $K$, Yánez $S$. Adaptación y validación de la Escala de Orientación a la Dominancia Social (SDO) en una muestra Chilena. Universitas Psychologica 2010;9:161-168.

16. Senra-Rivera C, De Arriba-Rossetto A, Seoane-Pesqueira G. Papel de la experiencia en la aceptación vs. Rechazo del paciente con esquizofrenia. Revista Latinoamericana Psicología 2008;40:73-83.

17. Thompson B. Exploratory and confirmatory factor analysis. Washington, DC: American Psychological Association; 2005.

18. Revelle W, Rocklin T. Very simple structure: An alternative procedure for estimating the optimal number of interpretable factor. Multivariate Behavioral Research 1979;14:403-414.

19. Hair J, Black W, Babin B, Anderson R. Multivariate data analysis. Séptima edición. New Jersey: Prentice-Hall; 2009.

20. Minoletti A. Plan nacional de salud mental en Chile. 10 años de experiencia. Rev Panam Salud Pública 2005;18:346-358.

21. Angermeyer M, Dietrich $S$. Public beliefs about and attitudes towards people with mental illness: a review of population studies. Acta Pychiatr Scand 2006;113:163-179.

22. Grausgruber A, Meise U, Katschning H, Schöny W et al. Patterns of social distance towards people suffering from schizophrenia in Austria: a comparison between the general public, relatives and mental staff. Acta Pychiatr Scand 2007;115:310-319.

23. De Toledo E, Blay SL. Community perception of mental disorders. A systematic review of Latin American and Caribbean studies. Soc Psychiatry Psychiatr Epidemiol 2004;39:955-961.

24. Hinshaw S. Stigma and mental illness: Developmental issues and future prospects. En: Cicchetti D, Cohen D (eds). Developmental psychopathology. Risk, disorder, and adaptation. Vol 3. New Jersey: John Wiley \& Sons; 2006.

25. Leiderman EA, Vazquez G, Berizzo C, Bonifacio A et al. Public knowledge, beliefs and attitudes towards patients with schizophrenia: Buenos Aires. Soc Psychiatry Psychiatr Epidemiol 2011;46:281-290. 